\title{
Palliative Care of the Patient with Advanced, Recurrent and Metastatic Cervical Cancer: An Institutional Study
}

\author{
Rajshree $\mathrm{DK}^{1 *}$, Mahesh $\mathrm{R}^{2}$ and Kothari $\mathrm{TO}^{3}$ \\ ${ }_{1}^{1}$ Professor, Department of Obstetrics and Gynecology, Cama and Albless Hospital, India \\ ${ }^{2}$ Asst Professor, Dept of Radiation Oncology, Cama and Albless Hospital, India \\ ${ }^{3}$ Senior Resident, Dept of Radiation Oncology, Cama and Albless Hospital, India
}

\section{Research Article}

Volume 3 Issue 2

Received Date: May 16, 2018

Published Date: June 28, 2018

*Corresponding author: Rajshree Dayanand Katke, Department of Obstetrics and Gynecology, Cama and Albless Hospital, Grant Govt Medical College and Sir JJ Groups of Hospitals, Mahanagar Palika Road, mumbai-01, India, Tel: 9869917830; Email: drrajshrikatke@gmail.com

\section{Abstract}

Introduction: Cancer of the female genital tract especially cervical cancer is a significant cause of morbidity and mortality worldwide. When potentially curative treatment options are unavailable or have proven ineffective, the clinical goal changes from cure to palliation.

Methodology: Retrospectively, 60 female patients were included in the study requiring palliative treatment for various symptoms.

Results: All patients were classified based on the symptoms they presented with, requiring palliative treatment.

Most patients presented with Pain, some form of metabolic derangement and GI symptoms needing attention. Bleeding, edemas were among the few other symptoms while fistulas, venous thrombosis and pulmonary complications were the least common ones.

Conclusion: Though Ca Cervix is vastly considered a curable disease now a days, palliative care still forms one of the most important arenas of the cancer management. The aim being improvement in the quality of life of the patients with treatment failures or advanced disease.

Keywords: Palliative Care; Patients; Treatment; Pain; Symptoms; Bleeding

\section{Introduction}

Cancer of the female genital tract is a significant cause of morbidity and mortality worldwide. In the US and in countries where Papanicolaou (Pap) smear screening and treatment of cervical dysplasia are widely implemented, ovarian cancer is responsible for more cancer deaths each year than cancers of the uterine corpus and cervix combined. Elsewhere, in the absence of effective screening and early intervention programs, cervical 
cancer is a much more common cause of gynecologic cancer death.

When potentially curative treatment options are unavailable or have proven ineffective, the clinical goal changes from cure to palliation. The various gynecologic cancers, although arising from anatomically adjacent organs, have different natural histories. Symptoms of progressive disease vary depending upon the site of primary tumor origin. Therefore, strategies to palliate disease progression are tailored to the complications caused by the particular combination of local invasion and distant spread encountered with tumors arising from a given site of origin [1-6].

\section{Methodology}

Sixty female patients with cancer of the cervix who presented with locally advanced disease or recurrent or metastatic disease were included in the study, retrospectively. Patients were classified based on the symptoms requiring palliative management. Most of the patients had more than one symptom.

\section{Findings at our Institute}

We studied 60 cases of Ca Cervix with locally advanced disease or having recurrence after primary management and also cases with metastatic disease, all requiring palliative care in some form or the other.

Following are the characteristics of the patients.

\begin{tabular}{|c|c|c|}
\hline \multirow{2}{*}{ Sr. No. } & Characteristic & $\begin{array}{c}\text { No of Patients } \\
(\mathbf{N}=60)\end{array}$ \\
\hline 1 & Mean Age & 46 \\
\hline \multirow{4}{*}{} & \multicolumn{2}{|c|}{ Symptoms } \\
\cline { 2 - 3 } & Bleeding PV & 20 \\
\cline { 2 - 3 } 2 & Pain & 45 \\
\cline { 2 - 3 } & Anemia & 17 \\
\cline { 2 - 3 } & Fistulas & 5 \\
\cline { 2 - 3 } & Edema & 16 \\
\cline { 2 - 3 } & Venous Thrombosis & 6 \\
\cline { 2 - 3 } & Pulmonary & $\mathbf{4 1}$ \\
\cline { 2 - 3 } & Metabolic & 34 \\
\cline { 2 - 3 } & Obstructive uropathy & 25 \\
\hline \multirow{4}{*}{3} & Gastro-Intestinal & 11 \\
\cline { 2 - 3 } & symptoms & 26 \\
\cline { 2 - 3 } & Locally Advanced & 23 \\
\cline { 2 - 3 } & Meco regional Recurrence & \\
\hline
\end{tabular}

Table1: characteristics of the patients.

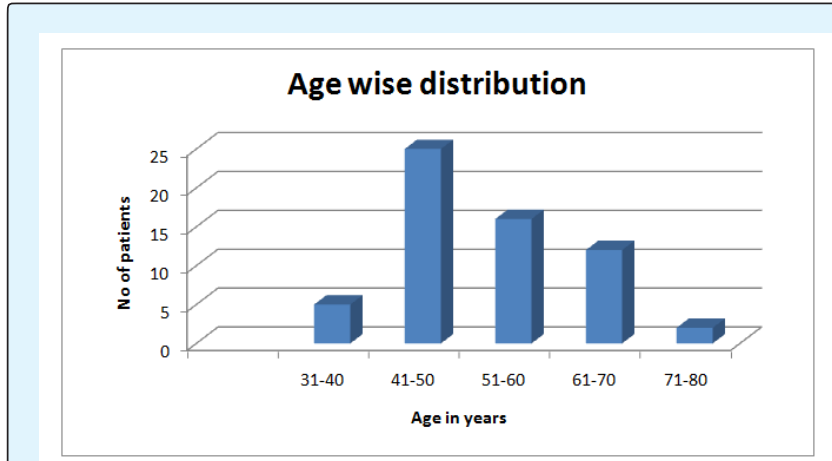

Figure 1: Age wise distribution.
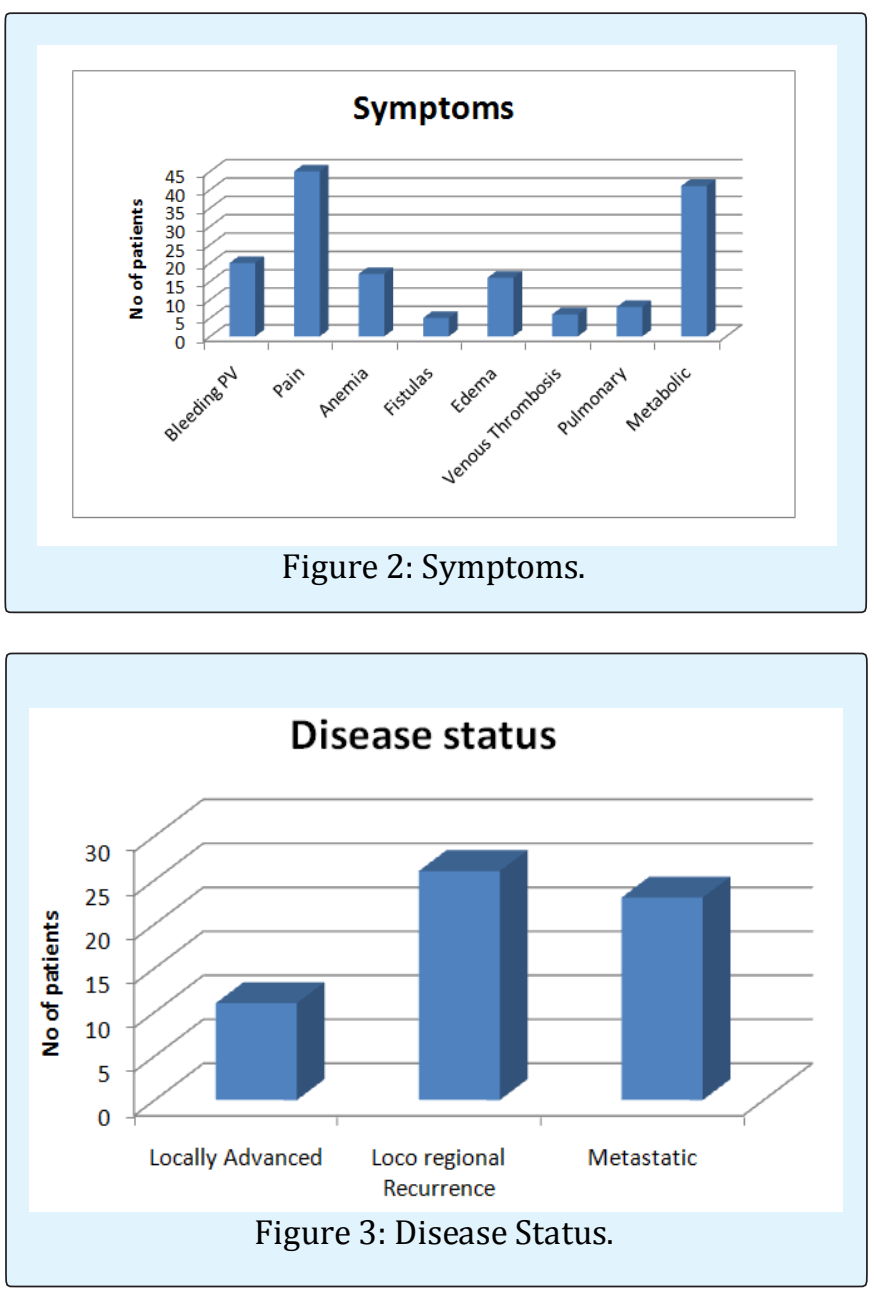

\section{Discussion}

\section{Bleeding \& Pain}

Cervical cancer tends to spread locally before it metastasizes to distant organs. When cervical cancer is confined to the pelvis or regional lymph nodes, it may be 
cured with radical surgery, chemo radiation, or both. When patients with cervical cancer have distant metastatic disease, the cancer generally is not curable. In this setting, any treatment administered is of palliative intent. As always, palliative treatment should be directed at symptom control. Patients with advanced or recurrent cervical cancer may have any of the following symptoms:

- Vaginal bleeding or discharge

- Pelvic or back pain

- Urinary or bowel fistulas

- Lower extremity edema

- Deep venous thrombosis

- Dyspnea from anemia or pulmonary involvement

- Uremia from ureteral obstruction

Available interventions to control vaginal bleeding include vaginal packing, radiation therapy, embolization of the uterine arteries, or surgical resection or arterial ligation. Vaginal packing is usually a temporary measure. Monsels solution (i.e., ferric sub sulfate) applied to the vaginal packing or even formalin applied to only the tip of the vaginal packing may enhance this temporary measure. Gauze, lamb's wool, or calcium alginate packing can be used. Potentially helpful radiation therapy approaches include transvaginal orthovoltage treatment, high dose fraction teletherapy, or brachytherapy.

Fulminant hemorrhage might require embolization of the uterine arteries, a procedure performed in the interventional radiology suite. If radiographically directed embolization is not available, another emergency alternative is laparotomy with ligation of the uterine arteries or the anterior divisions of the hypogastric arteries. A desperate measure of this intensity is not appropriate when widespread dissemination of disease causing imminent threat to the patient's life exists, but carefully selected patients may derive meaningful benefit. Symptomatic anemia from blood loss can be remedied with blood transfusions once cessation of bleeding is accomplished [7-12].

Pain is often a very disabling symptom of advanced or recurrent cervical cancer. Regional nerve, muscle, and bone infiltration can cause severe discomfort. Narcotic analgesics are a fundamental component of cancer pain treatment. Recognizing that narcotics can be delivered via many different routes is important. Agents may be prepared for oral, rectal, vaginal, sublingual, intravenous, intramuscular, epidural, and topical administration. Unfortunately, narcotics are associated with some troublesome common adverse effects that also must be addressed. These include constipation, pruritus, nausea, drowsiness, and skin rash. Because constipation is almost universal with increasing doses of narcotics, a bowel stimulant should be prescribed simultaneously.

Nonsteroidal anti-inflammatory analgesics and certain antidepressant medications often can provide a favorable synergistic effect when prescribed concurrently with narcotics, especially for pain suspected to be of neuropathic origin. When pain is directly attributable to specific foci of disease, such as bone metastasis or paraaortic lymph node recurrence, a brief course of palliative radiation therapy yields substantial pain reduction in a high percentage of patients. Transdermal electrical nerve stimulation (TENS), massage therapy, and meditation or other biofeedback techniques are sometimes helpful adjuncts to narcotic therapy.

Anxiety and depression are common co-morbidities in patients with malignancy of any type. Although these responses are not inappropriate in a patient diagnosed with a life-threatening condition, recognizing them and initiating intervention are important. Unless these conditions are treated adequately, patients might be noncompliant with other important therapies. Furthermore, efforts to control pain are particularly compromised. Fortunately, several effective medical therapies are available for both of these conditions. In addition to anxiolytics and antidepressants, supportive counseling, spiritual counseling, and family support can help counter feelings of depression and anxiety.

\section{Fistulas Edema and Venous Thrombosis}

Advanced cervical cancer may cause urinary fistulas, vesicovaginal fistulas more commonly, and, less commonly, ureterovaginal fistulas. Constant leakage of urine is extremely disturbing to many patients. Although not necessarily painful, fistulous drainage can have an extremely negative impact on quality of life. Patients with fistulas often may choose to avoid social and family encounters, ultimately becoming housebound.

Palliation of fistulas may be accomplished by either surgery to create an ureterointestinal conduit or by placing bilateral percutaneous nephrostomies and obstructing the ureters. Both procedures require an external appliance and maintenance. Functional status and operative risk should guide the selection of the means of palliation.

Although placement of nephrostomy tubes is a simpler procedure than surgical diversion of ureteral outflow, it is not necessarily a better choice for patients with a life 
expectancy of more than a few months. One disadvantage of percutaneous nephrostomies is the relative ease for these tubes to become kinked or dislodged. The tubes can be a source of infection and must be exchanged every few months. The use of external pads (diapers) to absorb drainage is the simplest option of all. However, in this authors experience, unless the patient is confined to bed for other reasons, this is a choice of minimal benefit for most patients.

Occasionally, rectovaginal fistulas occur from primary tumor invasion of the adjacent rectum. More often, these fistulas result from radiation injury or tumor recurrence. Diverting colostomy is the surgical procedure of choice in someone with a limited lifespan. Diverting end colostomy is associated with less long-term complications than loop colostomy.

Edema may result from generalized anasarca from protein depletion and malnutrition. Alternatively, edema may be localized to the lower extremities as a consequence of lymphatic and/or venous obstruction due to a large tumor burden in the lymph nodes. Symptomatic relief of edema and leg discomfort may be obtained by the use of graded compression stockings, elevation of the extremities, and diuretics. Physical therapists with training and expertise in lymphedema management can facilitate fluid drainage with external massage maneuvers.

Deep venous thrombosis (DVT) may cause secondary edema. For DVT developing for any other reason, anticoagulation is standard treatment unless medically contraindicated. Conventional or low molecular heparin usually is followed by oral warfarin. Prolonged anticoagulation is usually necessary because DVT often recurs in terminally ill patients with recurrent cancer. Anticoagulation prevents further extension of the thrombus and promotes gradual recanalization of the vessel as the thrombus is resorbed. At the same time, collateral vessels enlarge to accommodate more flow, and the net result is relief of extremity swelling and improved comfort for the patient. Because anticoagulation might exacerbate hemorrhage from recurrent cancer in the pelvis or elsewhere, in some cases, vena caval filters are preferable to prevent pulmonary emboli [13-17].

\section{Pulmonary Metabolic Gi}

Dyspnea may be a symptom of anemia, pleural effusion, infection, heart failure, or lymphangitic spread of cancer. Blood transfusions rapidly ameliorate the dyspnea of anemia. Thoracentesis with pleurodesis should improve the symptoms of a malignant pleural effusion.
Drainage of fluid is followed by pleural instillation of talc or doxycycline to sclerose the pleural lining. Pneumonia and heart failure should be treated as in the patient without cancer. Lymphangitic spread of cancer can cause hypoxia and dyspnea. Both oxygen and narcotics ameliorate this symptom.

Progressive or recurrent cervical cancer may cause uremia secondary to ureteral obstruction. Nausea, vomiting, somnolence, confusion, and seizures may result from uremia. Untreated uremia eventually is fatal. Death may be delayed if ureteral obstruction is relieved, either by percutaneous nephrostomy or ureteral stents.

Patients often request life-prolonging interventions as long as quality of life can be maintained through adequate pain control and other symptom relief. If other intercurrent complications of disease progression have proven refractory to medical or surgical intervention, then relieving ureteral obstruction to provide transiently improved excretion of uric acid and other waste products only prolongs the patient's pain and suffering. Patient and family counseling are necessary to identify the point at which further medical intervention is inappropriate in this setting.

Nausea and vomiting can occur as a result of mechanical obstruction of the small or large bowel. Metabolic derangements, such as uremia, infection, or central nervous system metastases, also can cause nausea. Vomiting from small bowel obstruction either can be relieved by small bowel resection and reanastomosis, bowel bypass, ileostomy, placement of a percutaneous gastrostomy tube, or nasogastric tube. Colonic obstruction usually occurs at the rectum or sigmoid. Transverse loop colostomy is a fast and relatively easy way to circumvent vomiting from this problem.

Metabolic causes of nausea and vomiting can be relieved by correcting the metabolic imbalance. Hypercalcemia is an uncommon paraneoplastic manifestation of metastatic gynecologic cancer. Hydration, diuretics, steroids, calcium-binding agents, and bisphosphonates should be considered. Immediate symptomatic relief of nausea may be obtained with the use of phenothiazines, antihistamines, steroids, or 5HT-3 antagonists. Nausea and vomiting caused by brain metastases can be improved by the use of radiation therapy and steroids.

Diarrhea also can accompany advanced or recurrent cervical cancer. While loose bowel movements are a frequent acute lower gastrointestinal toxicity from pelvic 
radiotherapy, this effect nearly always resolves within a few weeks after treatment is completed. Agents that reduce diarrhea include anticholinergics and opiate derivatives such as loperamide, codeine, diphenoxylate sodium with atropine, Kaopectate, paregoric, cholestyramine, and Donnatal.

Occasionally, diarrhea remains a long-term adverse effect following successful treatment of cervix cancer. Especially when patients experience exacerbation with intake of fatty foods, a suspected contributing influence is chronic mucosal change within the terminal ileum, where bile acid reabsorption can be impaired. Dietary modification can be particularly helpful in this regard.

\section{Conclusion}

Cervical cancer, despite being potentially preventable, remains an important cause of morbidity and gynecological cancer deaths throughout the world. The natural history is well understood, and randomized trials have established the optimal treatment strategies for patients with potentially curable disease at initial diagnosis. The management of patients with recurrent or metastatic disease has not been subjected to the same degree of investigation, and there are relatively few randomized trials to guide treatment decision-making especially from the palliative care point of view. The aim of this paper is to highlight the palliative care needs of the patients with advanced and recurrent cervical cancer.

\section{References}

1. Petterson F (1991) FIGO annual report on the results of treatment in gynecological cancer. Int J Gynecol Obstet 36: 27-130.

2. Perez CA, Camel HM, Kuske RR, Ming Shian K, Andrew $G$, et al. (1986) Radiation therapy alone in the treatment of carcinoma of the uterine cervix: A 20year experience. Gynecol Oncol 23(2): 127-140.

3. Montana GS, Fowler WC, Varia MA, Walton LA, Mack Y, et al. (1986) Carcinoma of the cervix, stage III: Results of radiation therapy. Cancer 57(1): 148-154.

4. Benedet J, Odicino F, Maisonneuve P (1998) Carcinoma of the cervix uteri: FIGO annual report on the results of treatment in gynaecological cancer. J Epidemiol Biost 3: 5-34.

5. Kim DS, Moon H, Kim KT, Hwang YY, Cho SH, et al. (1989) Two-year survival: Preoperative adjuvant chemotherapy in the treatment of cervical cancer stages IB and II with bulky tumor. Gynecol Oncol 33(2): 225-230.

6. Sardi J, Sananes C, Giaroli A, Maya G, di Paola G (1990) Neoadjuvant chemotherapy in locally advanced carcinoma of the cervix. Gynecol Oncol 38(3): 486493.

7. Eddy GL, Manetta A, Alvarez RD, Williams L, Creasman WT (1995) Neoadjuvant chemotherapy with vincristine and cisplatin followed by radical hysterectomy and pelvic lymphadenectomy for FIGO stage IB bulky cervical cancer: A Gynecologic Oncology Group pilot study. Gynecol Oncol 57(3): 412-416.

8. Namkoong SE, Park JS, Kim JW, Bae SN, Han GT, et al. (1995) Comparative study of the patients with locally advanced stages I and II cervical cancer treated by radical surgery with and without preoperative adjuvant chemotherapy. Gynecol Oncol 59(1): 136142.

9. Leone B, Vallejo C, Perez J, Cuevas MA, Machiavelli M, et al. (1996) Ifosfamide and cisplatin as neoadjuvant chemotherapy for advanced cervical carcinoma. Am J Clin Oncol 19(2): 132-135.

10. Kim SJ, Namkoong SE, Kim JH (1997) Clinical response to "quick cisplatin and etoposide" as neoadjuvant chemotherapy and its outcome in the uterine cervical cancer patients of stage IB2-IIIB. Proc Int Gynecol Cancer Soc 7: 38.

11. Zanetta G, Lissoni A, Pellegrino A, Sessa C, Colombo N, et al. (1998) Neoadjuvant chemotherapy with cisplatin, ifosfamide and paclitaxel for locally advanced squamous-cell cervical. Ann Oncol 9(9): 977-980.

12. Benedetti Panici P, Scambia G, Greggi S, Di Roberto P, Baiocchi G, et al. (1988) Neoadjuvant chemotherapy and radical surgery in locally advanced cervical cancer: A pilot study. Obstet Gynecol 71(3): 341-348.

13. Benedetti Panici P, Greggi S, Scambia G, Guglielmo R, Gabriela B, et al. (1991) High-dose cisplatin and bleomycin neoadjuvant chemotherapy plus radical surgery in locally advanced cervical cancer: A preliminary report. Gynecol Oncol 41(3): 212-216.

14. Panici PB, Scambia G, Baiocchi G, Greggi S, Ragusa G, et al. (1991) Neoadjuvant chemotherapy and radical 
surgery in locally advanced cervical cancer. Cancer 67(2): 372-379.

15. Benedetti-Panici P, Greggi S, Scambia G, Amoroso M, Salerno MG, et al. (1998) Long-term survival following neoadjuvant chemotherapy and radical surgery in locally advanced cervical cancer. Eur J Cancer 34(3): 341-346.

16. Giaroli A, Sananes C, Sardi J, Maya AG, Bastardas ML, et al. (1990) Lymph node metastases in carcinoma of the cervix uteri: Response to neoadjuvant chemotherapy and its impact on survival. Gynecol Oncol 39(1): 34-39.

17. Sananes C, Giaroli A, Soderini A, Guardado N, Snaidas L, et al. (1998) Neoadjuvant chemotherapy followed by radical hysterectomy and preoperative adjuvant chemotherapy in the treatment of the cervix uteri: Long-term follow-up of a pilot study. Eur J Gynecol Oncol 19(4): 368-373. 